\title{
Clinical features and practice patterns of treatment for adrenal crisis: a nationwide cross-sectional study in Japan
}

\author{
Yosuke Ono', Sachiko Ono², Hideo Yasunaga², Hiroki Matsui ${ }^{2}$, Kiyohide Fushimi ${ }^{3}$ \\ and Yuji Tanaka'
}

1Department of General Medicine, National Defense Medical College, Tokorozawa, Saitama, Japan, ${ }^{2}$ Department of Clinical Epidemiology and Health Economics, School of Public Health, The University of Tokyo, Bunkyo-ku, Tokyo, Japan, and ${ }^{3}$ Department of Health Policy and Informatics, Tokyo Medical and Dental University Graduate School of Medicine, Bunkyo-ku, Tokyo, Japan

Correspondence should be addressed to $\mathrm{Y}$ Ono Email

onoyousuke1979@yahoo.co.jp

\begin{abstract}
Context: Adrenal crisis is an endocrine emergency that requires prompt diagnosis and treatment. However, the clinical features and practice patterns of treatment for adrenal crisis are not completely understood.

Objective: To investigate patient characteristics, comorbidities and treatments of adrenal crisis.

Methods: We conducted a cross-sectional study of patients who received intravenous glucocorticoids for adrenal crisis at admission from 1 July 2007 to 31 March 2014, using a national inpatient database in Japan.

Results: Among approximately 34 million inpatients in the database, we identified 799 patients diagnosed with adrenal crisis and coexisting primary or secondary adrenal insufficiency at admission. The median (interquartile range) age was 58 (28-73) years, and the overall in-hospital mortality was $2.4 \%$ (19 of 799 patients). The most common comorbidity at admission was infections excluding pneumonia and gastroenteritis (15.0\%). There were $68(8.5 \%)$ patients with gastroenteritis, and no deaths occurred among these patients. The patients with secondary adrenal insufficiency showed significantly higher proportions of admission to ICU, extracellular fluid resuscitation, insulin therapy and catecholamine use than the patients with primary adrenal insufficiency. There were no significant between-group differences in mortality rate and variation in intravenous glucocorticoids (short-acting glucocorticoid, hydrocortisone; moderate-acting glucocorticoid, prednisolone or methylprednisolone; long-acting glucocorticoid, dexamethasone or betamethasone). Of the 19 dead patients, 15 were aged above 60 years, 12 had impaired consciousness at admission and 13 received insulin therapy.
\end{abstract}

Conclusions: Clinicians should be aware that older patients with impaired consciousness and diabetes mellitus are at relatively high risk of death from adrenal crisis.

\section{Introduction}

Adrenal crisis is a life-threatening emergent manifestation of adrenal insufficiency that requires prompt management $(1,2,3,4)$. Currently, glucocorticoids are widely used in patients with various inflammatory or autoimmune diseases, as well as with primary or secondary adrenal insufficiency (5). Long-term glucocorticoid therapy leads to hypothalamic-pituitary-adrenal axis suppression, resulting in adrenal crisis, when insufficient endogenous
(C) 2017 European Society of Endocrinology Printed in Great Britain glucocorticoids are produced to meet the demand of physical or emotional stress $(1,2,3,4)$.

Adrenal crisis is characterized by several nonspecific symptoms such as hypotension, hypovolemia, diarrhea, vomiting, abdominal pain, severe fatigue and hypoglycemia $(1,2,3,4)$. However, validated diagnostic criteria for adrenal crisis have not been established owing to its rarity $(1,2,3,4)$. In previous studies, adrenal 
crisis was defined as acute impairment of the general condition requiring hospital admission and intravenous glucocorticoid administration with or without fluid resuscitation $(6,7,8,9,10)$. The most common precipitating factor for adrenal crisis was infectious gastroenteritis because of insufficient oral glucocorticoid absorption disturbed by diarrhea and vomiting $(1,3,4,6$, $7,8,9,11)$. Medications such as barbiturates, rifampicin and mitotane can also trigger adrenal crisis $(1,6,12,13)$.

The current standard treatment for adrenal crisis is intravenous administration of hydrocortisone together with appropriate fluid resuscitation including $0.9 \%$ saline $(1,2,3,4)$. Prednisolone can be an alternative if hydrocortisone is unavailable (3). Although there have been several reports regarding the incidence, mortality, risk factors and prevention strategies of adrenal crisis $(6,7,8,9,10,11,14,15,16,17)$, no studies have focused on describing the clinical practice patterns for treatment of this endocrine emergency owing to the lack of suitable databases. Furthermore, the differences in clinical features of adrenal crisis between primary and secondary adrenal insufficiency remain to be investigated.

To address these issues, the aims of the present study were to describe the clinical features and practice patterns of treatment for adrenal crisis including supportive measures, based on a national inpatient database in Japan.

\section{Methods}

\section{Data source}

The Diagnosis Procedure Combination (DPC) database is a national inpatient database including discharge abstract and administrative claims data in Japan. The data were collected for 6 months per year from 1 July to 31 December in the fiscal years 2007-2009, for 9 months per year from 1 July to 31 March in the fiscal year 2010, and for 12 months per year from 1 April to 31 March since the fiscal year 2011. The number of inpatient admissions in the fiscal year 2013 was approximately 7 million in 1061 hospitals and represented more than $50 \%$ of all inpatient admissions to acute-care hospitals in Japan. The database has been utilized for various fields of clinical research $(18,19,20)$. The diagnoses of diseases are accurately recorded by the attending physicians. The database includes the following data: patients' sex and age; primary and secondary diagnoses; comorbidities at admission; admission to intensive care unit (ICU); medications and procedures; Japan Coma Scale (JCS) at admission and discharge status (alive or dead). Diagnoses are recorded using International Classification of Diseases Tenth Revision (ICD-10) codes and text data in Japanese. The JCS scores comprise consciousness levels of 0 (alert consciousness), 1-3 (drowsy: wakefulness without any stimuli), 10-30 (somnolent: arousal by some stimuli) and 100-300 (comatose) (21). The JCS scores are well correlated with the Glasgow Coma Scale scores (22).

\section{Ethical statement}

The present study was approved by the Institutional Review Board at The University of Tokyo. Written informed consent was not required because of the anonymous nature of the data.

\section{Patient selection}

From the database, we retrospectively extracted the records for all patients who were diagnosed as adrenal crisis (ICD code, E27.2) with coexisting primary or secondary adrenal insufficiency at admission and received intravenous glucocorticoids from 1 July 2007 to 31 March 2014. Patients with a suspected diagnosis of adrenal crisis were excluded, because we considered that their data may not be well validated owing to the lack of detailed clinical information, such as symptoms, signs and biochemical findings, in the database.

\section{Variables and main outcomes}

We investigated patients' sex and age, JCS scores at admission, medications and requirements for supportive measures. Age was categorized into $<20,20-39,40-59$, $60-79$ and $\geq 80$ years. Etiology of adrenal insufficiency was categorized into the following two groups: (i) primary adrenal insufficiency (A18.7, tuberculous Addison disease; E24.1, Nelson syndrome; E25.0, congenital adrenogenital disorders associated with enzyme deficiency; E25.8, idiopathic adrenogenital disorder; E25.9, adrenogenital syndrome; E27.1, primary adrenocortical insufficiency; E27.3, drug-induced adrenocortical insufficiency; E27.4, other and unspecified adrenocortical insufficiency; E27.8, other specified disorders of adrenal gland (congenital adrenal hyperplasia as the Japanese-text diagnosis in the DPC database); E71.3, adrenoleukodystrophy; E89.6, postprocedural adrenocortical hypofunction; Q89.1, congenital malformations of adrenal gland); 
Table 1 Clinical features of patients with adrenal crisis. Data are shown as $n(\%)$. All $P$ values were obtained by chi-square tests or Fisher's exact tests.

\begin{tabular}{|c|c|c|c|c|}
\hline & Overall $(n=799)$ & $\begin{array}{c}\text { Primary adrenal } \\
\text { insufficiency }(n=248)\end{array}$ & $\begin{array}{c}\text { Secondary adrenal } \\
\text { insufficiency }(n=551)\end{array}$ & $\boldsymbol{P}$ \\
\hline In-hospital death & $19(2.4)$ & $9(3.6)$ & $10(1.8)$ & 0.12 \\
\hline Sex & & & & 0.71 \\
\hline Male & 395 (49.4) & $125(50.4)$ & $270(49.0)$ & \\
\hline Female & $404(50.6)$ & $123(49.6)$ & $281(51.0)$ & \\
\hline Age (years) & & & & $<0.001$ \\
\hline$<20$ & $164(20.5)$ & $106(42.7)$ & $58(10.5)$ & \\
\hline $20-39$ & $102(12.8)$ & $36(14.5)$ & $66(12.0)$ & \\
\hline $40-59$ & $156(19.5)$ & $21(8.5)$ & $135(24.5)$ & \\
\hline $60-79$ & $280(35.0)$ & $57(23.0)$ & $223(40.5)$ & \\
\hline$\geq 80$ & $97(12.1)$ & $28(11.3)$ & $69(12.5)$ & \\
\hline JCS score at admission & & & & 0.094 \\
\hline 0 (alert) & $562(70.3)$ & $184(74.2)$ & $378(68.6)$ & \\
\hline $1-3$ (drowsy) & $137(17.1)$ & $42(16.9)$ & $95(17.2)$ & \\
\hline 10-30 (somnolent) & $55(6.9)$ & $9(3.6)$ & $46(8.3)$ & \\
\hline 100-300 (comatose) & $45(5.6)$ & $13(5.2)$ & $32(5.8)$ & \\
\hline \multicolumn{5}{|c|}{ Hormone replacement therapy } \\
\hline Thyroid hormone & $308(38.5)$ & $26(10.5)$ & $282(51.2)$ & $<0.001$ \\
\hline Vasopressin & $129(16.1)$ & $8(3.2)$ & $121(22.0)$ & $<0.001$ \\
\hline Growth hormone & $21(2.6)$ & $1(0.4)$ & $20(3.6)$ & 0.007 \\
\hline Testosterone & $10(1.3)$ & $0(0.0)$ & $10(1.8)$ & 0.036 \\
\hline
\end{tabular}

JCS, Japan Coma Scale; Thyroid hormone, levothyroxine sodium hydrate, liothyronine sodium or dried thyroid.

(ii) secondary adrenal insufficiency (E23.0, hypopituitarism; E23.6, other disorders of pituitary gland; E23.7, disorder of pituitary gland, unspecified; E89.3, postprocedural hypopituitarism). Hormone replacement therapy included thyroid hormone (levothyroxine sodium hydrate, liothyronine sodium and dried thyroid), vasopressin, growth hormone and testosterone. Medications that could possibly trigger adrenal crisis included inhibitors of adrenocortical steroid synthesis (mitotane, trilostane metyrapone), rifampicin and barbiturate. We identified the number of repeat hospitalizations.

We identified the following comorbidities at admission: gastroenteritis; cardiovascular diseases; respiratory diseases including pneumonia; renal diseases; neuromuscular diseases; infections excluding pneumonia and gastroenteritis; rheumatic diseases; gastrointestinal diseases excluding gastroenteritis; hepatopancreatobiliary diseases; psychiatric diseases; cerebrovascular diseases; dementia; malignancy and trauma.

Table 2 Comorbidities at admission in patients with adrenal crisis. Data are shown as $n(\%)$. All $P$ values were obtained by chi-square tests or Fisher's exact tests.

\begin{tabular}{|c|c|c|c|c|}
\hline & Overall $(n=799)$ & $\begin{array}{c}\text { Primary adrenal } \\
\text { insufficiency }(n=248)\end{array}$ & $\begin{array}{c}\text { Secondary adrenal } \\
\text { insufficiency }(n=551)\end{array}$ & $\boldsymbol{P}$ \\
\hline Infections & $120(15.0)$ & $45(18.1)$ & $75(13.6)$ & 0.097 \\
\hline Respiratory diseases & $94(11.8)$ & $30(12.1)$ & $64(11.6)$ & 0.85 \\
\hline Gastroenteritis & $68(8.5)$ & $32(12.9)$ & $36(6.5)$ & 0.003 \\
\hline Cardiovascular diseases & $51(6.4)$ & $13(5.2)$ & $38(6.9)$ & 0.38 \\
\hline Gastrointestinal diseases & $46(5.8)$ & $16(6.5)$ & $30(5.4)$ & 0.57 \\
\hline Neuromuscular diseases & $45(5.6)$ & $10(4.0)$ & $35(6.4)$ & 0.19 \\
\hline Renal diseases & $36(4.5)$ & $12(4.8)$ & $24(4.4)$ & 0.76 \\
\hline Malignancy & $27(3.4)$ & $12(4.8)$ & $15(2.7)$ & 0.13 \\
\hline Psychiatric diseases & $26(3.3)$ & $5(2.0)$ & $21(3.8)$ & 0.19 \\
\hline Cerebrovascular diseases & $25(3.1)$ & $5(2.0)$ & $20(3.6)$ & 0.23 \\
\hline Hepatopancreatobiliary diseases & $24(3.0)$ & $7(2.8)$ & $17(3.1)$ & 0.84 \\
\hline Rheumatic diseases & $10(1.3)$ & $3(1.2)$ & 7 (1.3) & 1.00 \\
\hline Dementia & $8(1.0)$ & $1(0.4)$ & $7(1.3)$ & 0.45 \\
\hline Trauma & $5(0.6)$ & $2(0.8)$ & $3(0.5)$ & 0.65 \\
\hline
\end{tabular}


We investigated the practice patterns for treating adrenal crisis in terms of variation in intravenous glucocorticoid therapy (short-acting glucocorticoid, hydrocortisone; moderate-acting glucocorticoid, prednisolone or methylprednisolone; long-acting glucocorticoid, dexamethasone or betamethasone). We also examined the use of mineralocorticoid (fludrocortisone), extracellular fluid (normal saline or Ringer's solution), intravenous glucose, glucagon, insulin, catecholamines (dopamine, noradrenaline, adrenaline and dobutamine), immune globulin, albumin, antithrombin and blood transfusion (red blood cells, fresh-frozen plasma and platelets). Supportive measures included mechanical ventilation and hemodialysis (i.e. intermittent or continuous renal replacement therapy).

\section{Statistical analysis}

We compared the patient characteristics between the two groups with primary and secondary adrenal insufficiency using the chi-square test or Fisher's exact test as appropriate. All tests were two-tailed, and $P<0.05$ was considered statistically significant. All statistical analyses were performed using SPSS, version 22.0 (IBM).

\section{Results}

\section{Clinical features of patients with adrenal crisis}

Among approximately 34 million inpatient admissions during the study period, we identified 799 eligible patients. The clinical features of the patients in the subgroups are shown in Table 1 . The median (interquartile range) age in the overall, primary adrenal insufficiency and secondary adrenal insufficiency groups was 58 (28-73), 29 (7-68), and 61 (41-73) years respectively. The ratio of females to males was 1.02 . The overall in-hospital mortality was $2.4 \%$ (19 of 799 patients). The patients with primary adrenal insufficiency were more likely to be younger than the patients with secondary adrenal insufficiency. The proportions of patients receiving thyroid hormone, vasopressin, growth hormone and testosterone were significantly higher in the patients with secondary adrenal insufficiency.

Table 3 Clinical practice patterns for adrenal crisis. Data are shown as $n(\%)$. All $P$ values were obtained by chi-square tests or Fisher's exact tests.

\begin{tabular}{l}
\hline \\
\hline Admission to ICU \\
Variation in intravenous glucocorticoids \\
Short-acting glucocorticoid \\
Moderate-acting glucocorticoid \\
Long-acting glucocorticoid \\
Mineralocorticoid \\
Extracellular fluid \\
Intravenous glucose \\
Glucagon \\
Insulin therapy \\
Catecholamine use \\
Variation in catecholamines \\
Dopamine \\
Noradrenaline \\
Adrenaline \\
Dobutamine \\
Immune globulin \\
Albumin \\
Antithrombin \\
Blood transfusion \\
Red blood cells \\
Fresh-frozen plasma \\
Platelets \\
Mechanical ventilation \\
Hemodialysise \\
\hline
\end{tabular}

\begin{tabular}{c}
\hline Overall $(n=799)$ \\
\hline $42(5.3)$ \\
$736(92.1)$ \\
$89(11.1)$ \\
$35(4.4)$ \\
$76(9.5)$ \\
$717(89.7)$ \\
$328(41.1)$ \\
$8(1.0)$ \\
$147(18.4)$ \\
$83(10.4)$ \\
$56(7.0)$ \\
$27(3.4)$ \\
$29(3.6)$ \\
$15(1.9)$ \\
$12(1.5)$ \\
$25(3.1)$ \\
$12(1.5)$ \\
$26(3.3)$ \\
$10(1.3)$ \\
$17(2.1)$ \\
$34(4.3)$ \\
$11(1.4)$ \\
\end{tabular}

\begin{tabular}{c}
\hline $\begin{array}{c}\text { Primary adrenal } \\
\text { insufficiency }(n=248)\end{array}$ \\
\hline $7(2.8)$ \\
$230(92.7)$ \\
$26(10.5)$ \\
$13(5.2)$ \\
$70(28.2)$ \\
$195(78.6)$ \\
$106(42.7)$ \\
$0(0.0)$ \\
$31(12.5)$ \\
$16(6.5)$ \\
$9(3.6)$ \\
$7(2.8)$ \\
$7(2.8)$ \\
$2(0.8)$ \\
$5(2.0)$ \\
$5(2.0)$ \\
$3(1.2)$ \\
\\
$5(2.0)$ \\
$2(0.8)$ \\
$4(1.6)$ \\
$10(4.0)$ \\
$2(0.8)$ \\
\end{tabular}

\begin{tabular}{|c|c|}
\hline $\begin{array}{l}\text { Secondary adrenal } \\
\text { insufficiency }(n=551)\end{array}$ & $\boldsymbol{P}$ \\
\hline $35(6.4)$ & 0.039 \\
\hline $506(91.8)$ & 0.66 \\
\hline 63 (11.4) & 0.69 \\
\hline $22(4.0)$ & 0.43 \\
\hline $6(1.1)$ & $<0.001$ \\
\hline $522(94.7)$ & $<0.001$ \\
\hline $222(40.3)$ & 0.52 \\
\hline $8(1.5)$ & 0.063 \\
\hline $116(21.1)$ & 0.004 \\
\hline $67(12.2)$ & 0.014 \\
\hline $47(8.5)$ & 0.012 \\
\hline $20(3.6)$ & 0.56 \\
\hline $22(4.0)$ & 0.41 \\
\hline $13(2.4)$ & 0.17 \\
\hline $7(1.3)$ & 0.42 \\
\hline 20 (3.6) & 0.23 \\
\hline $9(1.6)$ & 0.76 \\
\hline $21(3.8)$ & 0.19 \\
\hline $8(1.5)$ & 0.73 \\
\hline $13(2.4)$ & 0.60 \\
\hline $24(4.4)$ & 0.83 \\
\hline $9(1.6)$ & 0.52 \\
\hline
\end{tabular}

ahydrocortisone; ${ }^{b}$ prednisolone or methylprednisolone; 'dexamethasone or betamethasone; ${ }^{\mathrm{d}}$ normal saline or Ringer's solution; eintermittent or continuous renal replacement therapy. 
In terms of medications possibly triggering adrenal crisis, three patients with primary adrenal insufficiency received inhibitors of adrenocortical steroid synthesis, one patient with secondary adrenal insufficiency received rifampicin and one patient with primary adrenal insufficiency received barbiturate. Among 248 patients with primary adrenal insufficiency, 20 (8.1\%) were hospitalized twice, 6 (2.4\%) were hospitalized three times and $4(1.6 \%)$ were hospitalized more than four times. Similarly, among 551 patients with secondary adrenal insufficiency, 48 (8.7\%) were hospitalized twice, 9 (1.6\%) were hospitalized three times and 5 (0.9\%) were hospitalized four times.

\section{Comorbidities at admission}

Infections were the most common comorbidity at admission, followed by respiratory diseases, gastroenteritis, cardiovascular diseases, gastrointestinal diseases and neuromuscular diseases (Table 2). The proportion of patients with gastroenteritis was significantly higher in the patients with primary adrenal insufficiency than that in the patients with secondary adrenal insufficiency. Overall, 16 patients had both infections and respiratory diseases, and 3 patients had both infections and gastrointestinal diseases.

\section{Clinical practice patterns for adrenal crisis}

The clinical practice patterns for adrenal crisis are shown in Table 3 . Overall, the rate of admission to ICU was $5.3 \%$ (42 of 799 patients). The proportions of patients with admission to ICU, extracellular fluid resuscitation, insulin therapy and catecholamine use were significantly higher in the patients with secondary adrenal insufficiency. In contrast, the patients with primary adrenal insufficiency were more likely to receive mineralocorticoid. Overall, 43 patients were treated with both short-acting and moderate-acting glucocorticoid, and 17 patients were treated with both short-acting and long-acting glucocorticoid. Among the 562 patients with alert consciousness (JCS score

Table 4 Details of the in-hospital deaths in 19 patients with adrenal crisis. Data are shown as $n(\%)$.

\begin{tabular}{|c|c|c|c|}
\hline & Overall $(n=19)$ & $\begin{array}{c}\text { Primary adrenal } \\
\text { insufficiency }(n=9)\end{array}$ & $\begin{array}{c}\text { Secondary adrenal } \\
\text { insufficiency }(n=10)\end{array}$ \\
\hline \multicolumn{4}{|l|}{ Sex } \\
\hline Male & $11(57.9)$ & $6(66.7)$ & $5(50.0)$ \\
\hline Female & $8(42.1)$ & $3(33.3)$ & $5(50.0)$ \\
\hline \multicolumn{4}{|l|}{ Age (years) } \\
\hline$<20$ & $2(10.5)$ & $2(22.2)$ & $0(0.0)$ \\
\hline 20-39 & $0(0.0)$ & $0(0.0)$ & $0(0.0)$ \\
\hline $40-59$ & $2(10.5)$ & $1(11.1)$ & $1(10.0)$ \\
\hline $60-79$ & $6(31.6)$ & $3(33.3)$ & $3(30.0)$ \\
\hline$\geq 80$ & $9(47.4)$ & $3(33.3)$ & $6(60.0)$ \\
\hline \multicolumn{4}{|l|}{ JCS score at admission } \\
\hline 0 (alert) & $7(36.8)$ & $4(44.4)$ & $3(30.0)$ \\
\hline 1-3 (drowsy) & $4(21.1)$ & $2(22.2)$ & $2(20.0)$ \\
\hline $10-30$ (somnolent) & $0(0.0)$ & $0(0.0)$ & $0(0.0)$ \\
\hline 100-300 (comatose) & $8(42.1)$ & $3(33.3)$ & $5(50.0)$ \\
\hline \multicolumn{4}{|l|}{ Hormone replacement therapy } \\
\hline Thyroid hormone ${ }^{a}$ & $5(26.3)$ & $1(11.1)$ & $4(40.0)$ \\
\hline Vasopressin & $4(21.1)$ & $2(22.2)$ & $2(20.0)$ \\
\hline Admission to ICU & $5(26.3)$ & $3(33.3)$ & $2(20.0)$ \\
\hline \multicolumn{4}{|l|}{ Variation in intravenous glucocorticoids } \\
\hline Short-acting glucocorticoid ${ }^{b}$ & $18(94.7)$ & $9(100.0)$ & $9(90.0)$ \\
\hline Moderate-acting glucocorticoid ${ }^{c}$ & $5(26.3)$ & $3(33.3)$ & $2(20.0)$ \\
\hline Long-acting glucocorticoid ${ }^{d}$ & $1(5.3)$ & $0(0.0)$ & $1(10.0)$ \\
\hline Intravenous glucose & $18(94.7)$ & $9(100.0)$ & $9(90.0)$ \\
\hline Insulin therapy & $13(68.4)$ & $6(66.7)$ & $7(70.0)$ \\
\hline Catecholamine use & $12(63.2)$ & $6(66.7)$ & $6(60.0)$ \\
\hline Mechanical ventilation & $11(57.9)$ & $5(55.6)$ & $6(60.0)$ \\
\hline Hemodialysis ${ }^{\mathrm{e}}$ & $3(15.8)$ & $2(22.2)$ & $1(10.0)$ \\
\hline
\end{tabular}

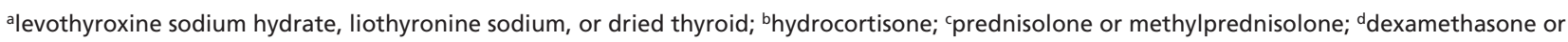

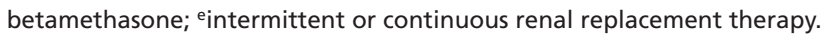


of 0 ) at admission, 19 were admitted to ICU, 35 were treated with catecholamines, 12 required mechanical ventilation and 4 required hemodialysis.

\section{Details of in-hospital deaths}

Details of the in-hospital deaths in 19 patients with adrenal crisis are shown in Table 4. Given the small sample size of dead patients, statistical comparisons were not performed. Among the 19 dead patients, 15 (78.9\%) were aged above 60 years, 12 (63.2\%) had impaired consciousness (i.e., JCS score above 1) at admission and 13 (68.4\%) received insulin therapy.

\section{Discussion}

We examined 799 patients with adrenal crisis using a national inpatient database in Japan. Our data suggest that the spectrum of adrenal crisis ranges widely from low to high risk. Furthermore, our results provide an overview of the practice patterns of treatment for this endocrine emergency. In particular, the proportions of older patients, patients with impaired consciousness at admission and patients with diabetes mellitus receiving insulin were relatively high among the dead patients. Overall, 92 patients (11.5\%) with adrenal crisis had experienced previous repeat admissions. These findings highlight the clinical importance of aging, consciousness level and coexisting other endocrine disorder, as well as the need for ongoing patient education to prevent adrenal crisis in the management of patients with adrenal insufficiency. To the best of our knowledge, the present study is the first of its kind to describe the treatment patterns, including intensive therapies, for adrenal crisis. The strength of our study, compared with previous studies, is the large number of patients with adrenal crisis evaluated.

The large difference in mean age between the two types of adrenal insufficiency may have arisen because primary adrenal insufficiency, such as autoimmune Addison's disease and congenital adrenal hyperplasia, is commonly caused by gene mutations (2), and thus, develops earlier in life. In contrast, the common causes of secondary adrenal insufficiency were reported to be pituitary adenoma and other intracerebral tumors $(6,11)$. We therefore consider that the patients with secondary adrenal insufficiency were more likely to develop their disease later in life than the patients with primary adrenal insufficiency.
In the present study, the overall in-hospital mortality of adrenal crisis was $2.4 \%$. In contrast, a recent prospective questionnaire survey in Germany showed that the adrenal crisis-related mortality of patients with primary or secondary adrenal insufficiency was 6.3\% (four patients in 64 episodes of adrenal crisis) (11). This discrepancy may be explained by the differences in sample size and study design between the previous questionnaire survey and the present study. Our study represents a real-world clinical setting based on a national database.

A previous study on adrenal crisis among inpatients aged above 20 years in Australia showed that the proportion of females was $62.5 \%$ (515 of 824 patients) (23). Several other studies also indicated that female patients with adrenal insufficiency were at higher risk for adrenal crisis $(6,11,14,15)$. In our study, the sex distribution of the patients with adrenal crisis was almost even, for unclear reasons.

Overall, approximately half of the patients with adrenal crisis were aged above 60 years, and older patients showed higher mortality. These findings highlight the importance of considering age when treating patients with adrenal insufficiency in the acute phase, because older patients are more likely to have several health problems and underlying comorbidities. For example, older patients with cognitive impairment may have difficulty in self-management of glucocorticoid stress doses when required (23). Moreover, older patients do not necessarily show typical symptoms of infectious diseases such as fever, and thereby delay self-management of glucocorticoid stress doses and intravenous administration of glucocorticoid by attending physicians. Thus, these problems can lead to the development of severe adrenal crisis with eventual lethality.

Patients with adrenal crisis and coexisting impaired consciousness at admission had relatively high mortality. One possible explanation is the existence of a point of no return in adrenal crisis, reflecting the point at which even optimum treatment will no longer prevent death from adrenal crisis (1). Consciousness level can be one of the criteria for determining the severity or grading of adrenal crisis. Notably, among the 19 dead patients, 7 patients $(36.8 \%)$ had alert consciousness (JCS score 0 ) at admission. We speculate that these patients became worse after admission because they required supportive care including catecholamine, mechanical ventilation and hemodialysis. These findings indicate that if the diagnosis of adrenal crisis is missed, even 
patients with alert consciousness in the early phases may possibly die.

Gastroenteritis, other infectious disease, diabetes insipidus and psychiatric disease were previously reported to be precipitating factors for adrenal crisis $(6,11,15)$. In our study, 68 (8.5\%) patients had gastroenteritis, and patients with primary adrenal insufficiency were more likely to have this comorbidity than patients with secondary adrenal insufficiency. No deaths were observed among the patients with gastroenteritis. We speculate that the patients with gastroenteritis were immediately treated with intravenous glucocorticoids because of apparent symptoms such as diarrhea and vomiting, resulting in prompt recovery. We further speculate that the diagnosis of gastroenteritis in adrenal crisis was potentially overestimated because the symptoms of this crisis, including nausea and vomiting, overlap with those of gastroenteritis and may be interpreted as acute gastroenteritis (23).

Interestingly, some patients with adrenal crisis were treated with moderate-acting or longacting glucocorticoid combined with short-acting glucocorticoid. The reasons for the use of moderateacting or long-acting glucocorticoid were not recorded in the database by the attending doctors. Previous European studies also described that some patients with adrenal insufficiency received prednisolone or dexamethasone (mostly combined with hydrocortisone, prednisolone or cortisone acetate) for unknown reasons $(6,8,11)$. Additional studies are needed to clarify how physicians use different types of glucocorticoid in patients with adrenal crisis.

Compared with the patients with primary adrenal insufficiency, the patients with secondary adrenal insufficiency were more likely to receive intensive therapies including admission to ICU, extracellular fluid resuscitation, insulin therapy and catecholamine use. These findings suggest that the patients with secondary adrenal insufficiency may have developed more severe adrenal crisis than the patients with primary adrenal insufficiency. However, there were no significant between-group differences in mortality rate and variation in intravenous glucocorticoids. Moreover, our findings cannot provide recommendations regarding the optimal treatment for adrenal crisis because of the small number of deaths and the retrospective study design.

Among the 19 dead patients, 13 received insulin, 5 received thyroid hormone and 4 received vasopressin.
These findings suggest that the patients with adrenal insufficiency and coexisting multiple endocrine disorders may have developed severe adrenal crisis. This interpretation is supported by previous evidence that patients with autoimmune polyglandular syndrome were at higher risk for developing adrenal crisis (24).

Differences in the definition of adrenal crisis among studies can lead to heterogeneous results and interpretations. As a top priority, internationally uniform and validated diagnostic criteria for adrenal crisis should be established to serve as a platform for the advancement of research in this field. Although the present study did not include detailed endocrinological data, we believe that our findings based on large-scale data will provide clues toward better understanding of the clinical features of this impending crisis.

Several limitations to the present study should be acknowledged. First, the diagnoses of adrenal crisis recorded in our study may be less well-validated than those in prospective studies, because the database did not include detailed clinical information such as symptoms, vital signs and laboratory data. Second, the clinical outcomes might have been affected by unrecorded confounding factors as follows: dose and duration of glucocorticoid administration before admission; whether or not patients discontinued taking glucocorticoid before admission; severity and duration of stressful events and whether or not patients were educated to manage sick days to avoid adrenal crisis, and were equipped with a steroid emergency card to inform medical practitioners of the need for immediate parenteral glucocorticoid administration. Third, we were unable to confirm the causal relationship between the patient characteristics in our study and higher mortality in adrenal crisis because of the nature of the cross-sectional study. Fourth, although the DPC database included more than $50 \%$ of all inpatient admissions to acute-care hospitals in Japan, population representativeness may be limited because the patient selection was not based on a random sampling method. Fifth, age may be a confounding factor that should be adjusted when comparing the two types of patients. However, we could not perform a multivariable logistic regression analysis to evaluate the risk factors associated with in-hospital mortality, owing to the small number of non-survivors. This is because the ratio of the number of non-survivors to the number of candidate independent variables (i.e., sex, age, consciousness level, admission to ICU, variation in intravenous glucocorticoids and catecholamine use) was small $(25,26)$. Finally, it was 
impossible to estimate the frequency of adrenal crisis in the study population with each etiology of adrenal insufficiency because the database did not include outpatient data. Despite these limitations, we believe that the present study serves to provide groundwork for future research in this field.

In conclusion, the present study suggests that clinicians should pay special attention to older patients with adrenal crisis and coexisting impaired consciousness and diabetes mellitus and treat them promptly and aggressively to prevent death from adrenal crisis. The necessary next steps are the establishment of internationally uniform and validated diagnostic criteria for adrenal crisis, and the establishment of a case registration system including detailed clinical information, with a view to achieving enhanced survival of this lethal endocrine emergency.

\section{Declaration of interest}

The authors declare that there is no conflict of interest that could be perceived as prejudicing the impartiality of the research reported.

\section{Funding}

This work was supported by Grants for Research on Policy Planning and Evaluation from the Ministry of Health, Labour and Welfare, Japan (grant numbers: H28-Policy-Designated-009 and H27-PolicyStrategy-011).

\section{References}

1 Allolio B. Extensive expertise in endocrinology. Adrenal crisis. European Journal of Endocrinology 2015172 R115-R124. (doi:10.1530/ EJE-14-0824)

2 Charmandari E, Nicolaides NC \& Chrousos GP. Adrenal insufficiency. Lancet 2014383 2152-2167. (doi:10.1016/S0140-6736(13)61684-0)

3 Bornstein SR, Allolio B, Arlt W, Barthel A, Don-Wauchope A, Hammer GD, Husebye ES, Merke DP, Murad MH, Stratakis CA et al. Diagnosis and treatment of primary adrenal insufficiency: an Endocrine Society clinical practice guideline. Journal of Clinical Endocrinology and Metabolism 2016101 364-389. (doi:10.1210/ jc.2015-1710)

4 Puar TH, Stikkelbroeck NM, Smans LC, Zelissen PM \& Hermus AR. Adrenal crisis: still a deadly event in the 21(st) century. American Journal of Medicine 2016129 339.e1-339.e9. (doi:10.1016/j.amjmed. 2015.08.021)

5 Marik PE \& Varon J. Requirement of perioperative stress doses of corticosteroids: a systematic review of the literature. Archives of Surgery 2008143 1222-1226. (doi:10.1001/archsurg. 143.12.1222)

6 Hahner S, Loeffler M, Bleicken B, Drechsler C, Milovanovic D, Fassnacht M, Ventz M, Quinkler M \& Allolio B. Epidemiology of adrenal crisis in chronic adrenal insufficiency: the need for new prevention strategies. European Journal of Endocrinology 2010162 597-602. (doi:10.1530/EJE-09-0884)
7 Reisch N, Willige M, Kohn D, Schwarz HP, Allolio B, Reincke M, Quinkler M, Hahner S \& Beuschlein F. Frequency and causes of adrenal crises over lifetime in patients with 21-hydroxylase deficiency. European Journal of Endocrinology 2012167 35-42. (doi:10.1530/EJE12-0161)

8 Smans LC, Van der Valk ES, Hermus AR \& Zelissen PM. Incidence of adrenal crisis in patients with adrenal insufficiency. Clinical Endocrinology 201684 17-22. (doi:10.1111/cen.12865)

9 White K \& Arlt W. Adrenal crisis in treated Addison's disease: a predictable but under-managed event. European Journal of Endocrinology 2010162 115-120. (doi:10.1530/EJE-09-0559)

10 Nagesser SK, van Seters AP, Kievit J, Hermans J, Krans HM \& van de Velde CJH. Long-term results of total adrenalectomy for Cushing's disease. World Journal of Surgery 200024 108-113. (doi:10.1007/s002689910020)

11 Hahner S, Spinnler C, Fassnacht M, Burger-Stritt S, Lang K, Milovanovic D, Beusclein F, Willenberg HS, Quinkler M \& Allolio B. High incidence of adrenal crisis in educated patients with chronic adrenal insufficiency: a prospective study. Journal of Clinical Endocrinology and Metabolism 2015100 407-416. (doi:10.1210/jc.2014-3191)

12 Elansary EH \& Earis JE. Rifampicin and adrenal crisis. British Medical Journal 1983286 1861-1862. (doi:10.1136/bmj.286.6381.1861-a)

13 Kyriazopoulou V, Parparousi O \& Vagenakis AG. Rifampicininduced adrenal crisis in addisonian patients receiving corticosteroid replacement therapy. Journal of Clinical Endocrinology and Metabolism 198459 1204-1206. (doi:10.1210/ jcem-59-6-1204)

14 Thompson SK, Hayman AV, Ludlam WH, Deveney CW, Loriaux DL \& Sheppard BC. Improved quality of life after bilateral laparoscopic adrenalectomy for Cushing's disease: a 10-year experience. Annals of Surgery 2007245 790-794. (doi:10.1097/01.sla.0000251578.03883.2f)

15 Omori K, Nomura K, Shimizu S, Omori N \& Takano K. Risk factors for adrenal crisis in patients with adrenal insufficiency. Endocrine Journal 200350 745-752. (doi:10.1507/endocrj.50.745)

16 Repping-Wuts HJ, Stikkelbroeck NM, Noordzij A, Kerstens M \& Hermus AR. A glucocorticoid education group meeting: an effective strategy for improving self-management to prevent adrenal crisis. European Journal of Endocrinology 2013169 17-22. (doi:10.1530/ EJE-12-1094)

17 Hahner S, Hemmelmann N, Quinkler M, Beuschlein F, Spinnler C \& Allolio B. Timelines in the management of adrenal crisis - targets, limits and reality. Clinical Endocrinology 201582 497-502. (doi:10.1111/cen.12609)

18 Ono Y, Ono S, Yasunaga H, Matsui H, Fushimi K \& Tanaka Y. Factors associated with mortality of thyroid storm: analysis using a national inpatient database in Japan. Medicine 201695 e2848. (doi:10.1097/ MD.0000000000002848)

19 Hamada T, Yasunaga H, Nakai Y, Isayama H, Horiguchi $H$, Matsuda S, Fushimi K \& Koike K. Continuous regional arterial infusion for acute pancreatitis: a propensity score analysis using a nationwide administrative database. Critical Care 201317 R214. (doi:10.1186/cc13029)

20 Ono S, Ishimaru M, Matsui H, Fushimi K \& Yasunaga H. Effect of hospital volume on outcomes of surgery for cleft lip and palate. Journal of Oral and Maxillofacial Surgery 201573 2219-2224. (doi:10.1016/j.joms.2015.04.003)

21 Todo T, Usui M \& Takakura K. Treatment of severe intraventricular hemorrhage by intraventricular infusion of urokinase. Journal of Neurosurgery 199174 81-86. (doi:10.3171/jns.1991.74.1.0081)

22 Ono K, Wada K, Takahara T \& Shirotani T. Indications for computed tomography in patients with mild head injury. Neurologia MedicoChirurgica 200747 291-298. (doi:10.2176/nmc.47.291)

23 Rushworth RL \& Torpy DJ. A descriptive study of adrenal crises in adults with adrenal insufficiency: increased risk with age and in 
those with bacterial infections. BMC Endocrine Disorders 20141479. (doi:10.1186/1472-6823-14-79)

24 Meyer G, Badenhoop K \& Linder R. Addison's disease with polyglandular autoimmunity carries a more than 2.5-fold risk for adrenal crises: German Health insurance data 2010-2013. Clinical Endocrinology 201685 347-353. (doi:10.1111/ cen.13043)
25 Harrell FE, Lee KL, Matchar DB \& Reichert TA. Regression models for prognostic prediction: advantages, problems, and suggested solutions. Cancer Treatment Reports 198569 1071-1076.

26 Peduzzi P, Concato J, Kemper E, Holford TR \& Feinstein AR. A simulation study of the number of events per variable in logistic regression analysis. Journal of Clinical Epidemiology 199649 1373-1379. (doi:10.1016/S0895-4356(96)00236-3)

Received 24 September 2016

Revised version received 15 December 2016

Accepted 10 January 2017 